\title{
UNA FACULTAD, UNA HISTORIA: DE LA PRÁCTICA AL DESARROLLO EPISTEMOLÓGICO DE LOS DOCENTES DE ENFERMERÍA DE UNA UNIVERSIDAD PÚBLICA
}

\section{A FACULTY, A STORY: FROM PRATICE TO EPISTEMOLOGICAL DEVELOPMENT OF TEACHERS OF THE FACULTY OF NURSING OF A PUBLIC UNIVERSITY}

\author{
Margarita.Córdova ${ }^{1}$ \\ Heddy Sofía Manrique ${ }^{2}$ \\ Gladys Marcelina Puza ${ }^{3}$ \\ Universidad Nacional “San Luís Gonzaga” Perú
}

\section{RESUMEN}

El objetivo fue determinar en la facultad y 1 margaritacordova_2004@hotmail.com,https://orcid.org/0000-0002-5881-8134, Universidad Nacional "San Luís Gonzaga"

2 heddymanrique@hotmail.com, https://orcid. org/0000-0002-0987-8632, Universidad Nacional "San Luís Gonzaga”

3 puzamendoza_g@hotmail.com,https://orcid. org/0000-0003-43085713,Universidad Nacional "San Luís Gonzaga" en su historia: la práctica y el desarrollo epistemológico en docentes de la Facultad de Enfermería de una universidad pública, trabajo de investigación descriptivo, transversal con 40 docentes; los resultados fueron Edad promedio 58.6 años, $65 \%$ tienen especialidad, $87.5 \%$ maestría, $32.5 \%$ doctorado. Ejercicio profesional promedio 31 años; el 85\% decidieron ser enfermeros porque les gustaba la carrera. Mayoritariamente consideran suficiente la 
formación teórica y práctica que recibieron. Aplican modelos teóricos y conceptuales de enfermería, planes de cuidado, elaboran y publican trabajos. Consideran que el rol autónomo es característico de la profesión. Las concepciones epistemológicas priorizados en mayor proporción, fueron el cuidado individual, el proceso interactivo enfermera-paciente, satisfacción de las personas, la concepción integral y sistémica del paciente. En cuanto al ejercicio profesional, $87.5 \%$ están satisfechos, señalan que desde las áreas asistenciales, investigación y docencia, se realizan las aportaciones más significativas para la profesión. Mayoritariamente utilizan proceso de atención de enfermería, y con frecuencia paradigmas epistemológicos. Conclusiones: La práctica asistencial combinada con la docencia y la investigación, ha generado el desarrollo epistemológico de los docentes

PALABRAS CLAVE: Desarrollo epistemológico, Práctica de la enfermería, Facultad

\section{ABSTRACT}

The objective was to determine in the faculty and in its history: the practice and epistemological development in teachers of the Faculty of Nursing of a public university, descriptive, cross-sectional research work with 40 teachers; The results were Average age 58.6 years, $65 \%$ have a specialty, $87.5 \%$ a master's degree, $32.5 \%$ a doctorate. Average professional practice 31 years; $85 \%$ decided to be nurses because they liked the career. Most of them consider the theoretical and practical training they received sufficient. They apply theoretical and conceptual nursing models, care plans, elaborate and publish works. They consider that the autonomous role is characteristic of the profession. The epistemological conceptions prioritized in the greatest proportion were individual care, the nurse-patient interactive process, people's satisfaction, and the comprehensive and systemic conception of the patient. Regarding professional practice, $87.5 \%$ are satisfied, they point out that from the healthcare, research and teaching areas, the most significant contributions are made to the profession. They mostly use the nursing care process, and often epistemological paradigms. Conclusions: The care practice combined with teaching and research has generated the epistemological development of teachers

KEYWORDS: Epistemological development, Nursing practice, Faculty

\section{INTRODUCCIÓN}

La Epistemología es la parte de la filosofía que estudia los principios, fundamentos, extensión y métodos del conocimiento humano (1). Se dice que es la ciencia o teoría del conocimiento, sometido a una reflexión rigurosa, critica. Hay quienes afirman que son las circunstancias históricas, psicológicas, sociológicas, que llevan a la obtención del conocimiento y los criterios por los cuales se lo justifica o invalida, y que requiere de una definición clara y precisa de los conceptos epistémicos como la verdad, objetividad, realidad o justificación.

Cuando ejercemos la docencia transmitimos conocimientos experiencias vivencias a través del proceso de enseñanza-aprendizaje, que van madurando, cambiando, enriqueciéndose, actualizándose, tanto en la práctica asistencial como en la práctica docente, de la mano de nuestra formación y capacitación personal permanente, la investigación, la aplicación de concepciones epistemológicas, modelos, paradigmas, teorías, y satisfacción con el ejercicio profesional. Esto forma parte de la historia del conocimiento de los docentes de enfermería, y del desarrollo epistemológico.

Los desafíos actuales y futuros de la profesionalización de enfermería plantean la necesidad de desarrollar un proceso formativo sustentado en la idoneidad, excelencia y calidad, que permita a la enfermería situarse 
como una profesión autónoma dentro de las disciplinas que brindan cuidado para la salud (2). Este proceso obliga a crear modelos y teorías propias que sustenten la profesión, y a desarrollar investigaciones que incrementen el saber del propio campo disciplinar. De este modo, la enfermería para alcanzar su finalidad debe ofrecer conocimiento sobre el objeto de estudio, bases científicas que la sustenten, teorías y modelos que integren las bases del conocimiento, y debe fortalecerse con la investigación ${ }^{(3)}$.

Para reflexionar sobre el desarrollo epistemológico de Enfermería, se hace necesario partir de la revisión de la definición de epistemología. Ésta es la rama de la filosofía que considera la historia del conocimiento. Suscita y responde preguntas relacionadas con el origen, la naturaleza, los métodos y las limitaciones del desarrollo del conocimiento y describe los diversos criterios con los cuales éste se acepta. Por esta razón los aspectos epistemológicos contribuyen a aumentar la conciencia sobre la complejidad y diversidad de las perspectivas, enfoques y teorías en los progresos, la verdad y sus métodos; bajo esta perspectiva Meleis citado por Claudia Ariza Olarte. Desarrollo epistemológico de enfermería. 2011, hace distinción entre los tipos de problemas en el conocimiento y desarrollo, por esto busca aquellos que pueden parecer los más apropiados para el progreso teórico de una disciplina, en este caso, Enfermería; y maneja las restricciones epistemológicas potenciales aun cuando sean inadecuadas, que surgen de la aceptación de factor de enfoque, de una teoría o de una perspectiva, sin hacer un estudio cuidadoso de las alternativas (4). Este artículo tiene como fin reflexionar sobre el desarrollo epistemológico que ha tenido la enfermería hasta hoy; para ello, en primer lugar, se discutirá sobre los paradigmas y las visiones del desarrollo del conocimiento y la realidad opuesta; evolución del conocimiento y su jerarquización; y por último la importancia que tiene la utilización de este conocimiento en la práctica. Se espera que este artículo contribuya a una mejor comprensión de la disciplina y su relación con la práctica de enfermería, que el conocimiento existente pueda ser valorado por la comunidad de enfermería, y como resultado pueda ser útil para guiar a quienes trabajan en la asistencia, y en la enseñanza docente a nivel universitario con grado de licenciatura y de postgrados ${ }^{(5)}$.

La mayoría de los egresados de las diferentes Facultades y Escuelas Académicas del País de Enfermería prefieren trabajar en Lima o las capitales de regiones, ocasionando una sobreoferta en estas zonas y un déficit marcado en el interior del país. Esta sobreoferta ocasiona la precariedad en las condiciones de trabajo de estos profesionales. La salud de la población peruana es un reflejo de su realidad social sin embargo se viene haciendo grandes esfuerzos por mejorar los indicadores, con la participación activa y muy comprometida de los recursos humanos de salud con la finalidad de asegurar el acceso equitativo a servicios de calidad a toda la población, es preciso tener en cuenta que: “El RRHH constituye un elemento fundamental en la producción de servicios de salud tanto en la dimensión subjetiva relativa a la intencionalidad humana como en la dimensión objetiva de la ejecución de competencias profesionales específicas"

\section{ANTECEDENTES:}

María Rosario Verástegui León. “Significado del cuidado de enfermería otorgado por las enfermeras de un servicio de cirugía de un hospital de Chiclayo 2012" Chiclayo.2014. El referente teórico: Jean Watson con su teoría del cuidado humano, la trayectoria metodológica cualitativa descriptiva comprensiva con enfoque procesual a través de la teoría de las representaciones sociales de Serge Moscovici, que permitió comprender el proceso de 
construcción del cuidado de enfermería en la práctica profesional, en las experiencias cotidianas e intersubjetivas. Los resultados, que se pudo acceder a las representaciones y siendo el gran núcleo central el significado del cuidado otorgado por las enfermeras, formado por cuatro dimensiones: "Reconocimiento del ser cuidado como persona humana en la interrelación", "Reconocimiento de la propia naturaleza humana de la enfermera", "Auto reconocimiento de capacidades de la enfermera" y "Definiendo que el cuidado de enfermería no es solo ciencia y arte, es la complementación con el Ser de la enfermera". Estos significados confirman que en la interrelación enfermera -persona cuidada hay esencias y crecimiento mutuo, lo cual permite a las enfermeras sentirse reconfortadas, realizadas como profesionales ${ }^{(6)}$

\section{MARCO TEÓRICO:}

En el caso de Enfermería, el primer momento del desarrollo del conocimiento fue intuitivo; la experiencia frente al manejo de situaciones marchó fundamentada en el ensayo y error. Los conocimientos fueron transmitidos de una generación a otra mediante el lenguaje oral, convirtiéndose el cuidado en una vocación de ayuda y de servicio en la que se logra acumular una experiencia empírica y un quehacer que no se diferenciaba de otros oficios ${ }^{(7)}$ Posteriormente se siente la necesidad de buscar un fundamento teórico acorde con el desarrollo de otros conocimientos para prestar el cuidado. Se comienzan a gestar prácticas sanitarias orientadas al cuidado de las personas haciendo énfasis en las normas de higiene, de control del medio ambiente, hacia la observación de los pacientes y su comodidad, a la administración de medicamentos ordenados por el médico. En este momento se centra la práctica en el cumplimiento de un quehacer señalado por el médico, con poca autonomía frente al cuidado y un estricto cumplimiento de reglas, principios y tradiciones repetitivas que alejaban esta práctica de un quehacer profesional y lo reducían a un oficio, pero con un acúmulo importante de experiencia por los desarrollos empíricos con los que se contaba hasta ese momento. Este proceso evolutivo conduce a que se establezcan programas de formación estructurados que permitan una mejor fundamentación de las bases teóricas que enriquezcan la práctica de cuidado. Se establecen planes de estudio orientados hacia las ciencias básicas como biología, anatomía, fisiología, microbiología; estás eran ciencias ya desarrolladas. Así se da comienzo a un período de consolidación del conocimiento; éste se caracteriza porque la experiencia acumulada y el nuevo conocimiento forman una alianza que genera nuevas necesidades en el campo de la ética y de lo jurídico y así se conforman organizaciones que respaldan el ejercicio de la profesión. En esta evolución del conocimiento de enfermería se reconocen dos paradigmas: el empirista y el interpretativo. Ellos representan visiones del desarrollo del conocimiento y de la realidad opuesta. El empirismo se basa en supuestos de que lo que se conoce se puede verificar por los sentidos. La suposición ontológica del empirismo es que hay una realidad que está afuera en algún lugar y que se puede validar por los sentidos. En este paradigma el conocimiento se desarrolla por observación del mundo natural para verificar y justificar las teorías que describen, predicen y prescriben. En enfermería este paradigma es útil porque facilita que se compruebe y valide la relación de las teorías y si esto resulta válido éstas se pueden usar para explicar, predecir y prescribir principios que son esenciales e importantes para la práctica clínica ${ }^{(8)}$

Por otro lado está el paradigma interpretativo; se puede afirmar que éste evolucionó debido a que muchas enfermeras se formaron en disciplinas como filosofía, sociología y antropología y por esta razón vieron el mundo desde otros puntos de vista. Este paradigma se caracteriza por 
supuestos ontológicos de que la realidad es compleja, holística y dependiente del contexto. El enfoque de la investigación está sobre las experiencias humanas; se hace énfasis en la subjetividad y no en la objetividad únicamente. Teniendo en cuenta que la realidad y la experiencia humana son variables, se valoran las múltiples formas de descubrir el conocimiento derivado de la experiencia humana. Estos métodos interpretativos comprenden la fenomenología, la hermenéutica, la teoría fundamentada, la etnografía y otros.

La formación de enfermeras es una práctica compleja y multifuncional que desempeña distintas funciones con relación al sistema social y sanitario. Dada la complejidad de la formación universitaria en general y de enfermeras en particular y de los procesos que en ella se generan carece de sentido tratar de dar cuenta de ellos desde una sola perspectiva o recurriendo a explicaciones causales unilaterales. Ciertamente ningún planteamiento de los dos que a continuación se ofrecen es lo suficientemente amplio y potente para explicar en toda su extensión y complejidad la realidad educativa. Sin embargo, ello no significa que debamos aceptar acríticamente cualquier propuesta. Existen intentos marcos conceptuales para fundamentar la formación práctica en enfermerías más actuales que otros considerados como obsoletos

En síntesis, la formación de enfermeras no es una entelequia que se desarrolla de modo inconexo con el desarrollo de la investigación y el conocimiento pedagógico. Su concepción, comprensión y materialización derivan de las perspectivas conceptuales y marcos teóricos sobre la formación universitaria que son predominantes en un momento histórico concreto. Estos marcos contienen las definiciones de cuidado, salud, función profesional, currículo, enseñanza, investigación, etc., y, lógicamente, la de formación de enfermeras, las cuales informan y determinan la menudo en forma de contenido curricularl la formación de las futuras profesionales del cuidado.

Materiales Y Métodos. Trabajo de Investigación cuantitativo, descriptivo correlacional. La población lo constituyeron los docentes de la facultad de enfermería de la Universidad Nacional San Luis Gonzaga de Ica. La muestra fue de 40 docentes, quienes en forma voluntaria accedieron a proporcionar la información. Para recolectar la información de campo, se utilizó como técnica la encuesta e instrumento el cuestionario. Para el análisis de datos se utilizó la estadística descriptiva e inferencial.

\section{RESULTADOS.}

I. Caracterización de los Docentes - Facultad Enfermería. La edad promedio es de 58.6 años, en mayor proporción o el $40 \%$ tienen edades entre 52 y 60 años, el $97.5 \%$ son mujeres, $87.5 \%$ obtuvieron el grado de bachiller y el título profesional en universidades del País, el 65\% tienen título de segunda especialidad, $87.5 \%$ son magísteres, $32.5 \%$ son doctores. En promedio tienen 31 años de ejercicio profesional, de los cuales $42.5 \%$ registran entre 21 a 30 años de servicio. El promedio de años en la docencia universitaria es de 21 años. En promedio el ejercicio asistencial es de $28.9 \%$, de los cuales $47.5 \%$ prestan servicio asistencial entre 21 a 30 años. El $100 \%$ son docentes de la Facultad de Enfermería, 32.5\% laboran también en el hospital Santa María del Socorro, el $22.5 \%$ en ESSALUD y el $40 \%$ en el Hospital Regional de Ica, el $47.5 \%$ son docentes principales, $45 \%$ asociados, $5 \%$ auxiliares, el $87.5 \%$ prestan labor asistencial y el $7.5 \%$ en la supervisión en enfermería.

\section{FORMACIÓN PROFESIONAL}

\section{Decisión de estudiar enfermería.}

En cuanto a la decisión por estudiar enfermería, $65 \%$ de los docentes afirmaron que fue por 
vocación, $85 \%$ para ejercer la profesión que más le gustaba, $22.5 \%$ por ser estudios de corta duración, $27.5 \%$ por tener buenas salidas profesionales, $10 \%$ decidieron por tradición familiar, y $15 \%$ por considerar que eran estudios fácilmente superables.

\section{Característica de la profesión}

El $87.5 \%$ de los docentes consideran que una características importante de la profesión de enfermería es el rol autónomo relacionado con los cuidados de salud de la persona; $85 \%$ señalan como otra característica importante a las actividades interdependientes destinadas a contribuir al diagnóstico y tratamiento de la enfermedad; y $67.5 \%$ consideran importante las actividades dependientes del diagnóstico y tratamiento médico.

\section{CONCEPCIONES PRESENTES EN LA PRÁCTICA PROFESIONAL}

Persona: Consideraciones a la hora de planificar y aplicar los cuidados.

El $45 \%$ de los docentes consideran prioritario tener en cuenta al paciente, quien tiene componentes físicos, intelectuales, emocionales, culturales y espirituales, el $27.5 \%$ de los docentes consideran como segunda prioridad tener en cuenta que los pacientes tienen capacidad y responsabilidad de decidir sobre su situación y el $17.5 \%$ de los docentes consideran como tercera prioridad, tener en cuenta que los pacientes no tienen capacidad para participar en sus cuidados, cuando se requiere de tecnologías específicas para tal fin, y que el cuidado es holístico, espiritual y cultural .

Salud: Concepciones que se aportan sobre la salud.

El $45 \%$ de los docentes consideran como primera prioridad la concepción sobre el estado de equilibrio, armonía y estabilidad, deseable y percibido positivamente, y $20 \%$ consideran como segunda prioridad y el $17.5 \%$ de los docentes consideran como tercera prioridad la independencia, y la integridad física y homeostática.

Entorno: Aspectos considerados en el cuidado del paciente. El 47.5\% de los docentes consideraron prioritario, que el cuidado de enfermería está compuesto por todos los elementos externos que rodean a la persona, el $20 \%$ de los docentes consideran como segunda prioridad, saber que el cuidado de enfermería está determinado por aspectos físicos, sociales y culturales, está constituido por los contextos histórico, social, político y cultural, que está compuesto por un conjunto del universo del que la persona forma parte íntegramente y el $15 \%$ de los docentes consideran como tercera prioridad, saber que el cuidado de enfermería, está compuesto por un conjunto del universo del que la persona forma parte íntegramente.

\section{DATOS RELACIONADOS CON LA VALORACIÓN DEL EJERCICIO PROFESIONAL.}

Satisfacción con el ejercicio profesional .El 52.5\% de los docentes están muy satisfechos con el ejercicio de su profesión, 35\% están satisfechos, y $12.5 \%$ no están satisfechos ni insatisfechos con el ejercicio de su profesión.

Uso de paradigma epistemológico. El 52.5\% de los docentes, con frecuencia utilizan paradigma epistemológico mixto, $40 \%$ paradigma racionalista, y $22.5 \%$ empirista.

\section{DISCUSIÓN}

Caracterización De Los Docentes. En el trabajo de investigación "Aplicación Del Proceso Atención de Enfermería en México: Un Análisis Epistemológico". Trabajo terminal que para obtener el grado de: Maestra en Enfermería en Oncología presentado por Edith Fuentes Estrada. Toluca, México -2014, en relación al nivel de estudios de las entrevistadas se observa que la mayoría de las participantes cuentan 
con el grado de Maestría ${ }^{(9)}$ y la minoría se ubica en la Especialidad respectivamente. Las Instituciones donde laboran las enfermeras participantes fueron: Dependencias del Gobierno Federal, particularmente Secretaría de Salud, Instituciones Académicas, Hospitales de Sector Público y Privado, el cual coincide con la caracterización de los docentes entrevistados para el presente trabajo de investigación. ${ }^{(2)}$ Los trabajos de investigación concuerdan con la información obtenida.

Formación Profesional. Francisco del Rey, Cristina en su trabajo de investigación "De la práctica de la enfermería a la teoría enfermera. Concepciones presentes en el ejercicio profesional". Universidad de Alcalá- España. 2008; indica que de las enfermeras participantes en el estudio: Un (67\%) eligen estudiar enfermería por vocación; el $(73,7 \%)$ para poder ejercer la profesión que más les gusta; y por tener buenas salidas profesionales $(40,7 \%)$. Consideran adecuado al aprendizaje teórico el $64 \%$ y el práctico al $57,6 \%$, siendo quienes cuentan con 30 años y más de experiencia profesional las más satisfechas, cuyo trabajo coincide con información concluida en el presente trabajo. ${ }^{(10)}$

Concepciones Epistemológicas. Se ha observado confusión por delimitar el dominio profesional, con una marcada tendencia a considerar el rol interdependiente por delante del autónomo y a éste con la misma valoración que el dependiente. Al tiempo que se detecta una formación y conocimiento limitados de los aspectos epistemológicos de la enfermería. Este hecho repercute en la heterogeneidad existente sobre las concepciones de los fenómenos paradigmáticos, porque cuando se aprecia una mayor formación conceptual y metodológica, se observa una tendencia más homogénea al elegir las dimensiones otorgadas a cada uno de los conceptos paradigmáticos, concentrándose en menos dimensiones refiere Francisco Del Rey, Cristina "De la práctica de la enfermería a la teoría enfermera. Concepciones presentes en el ejercicio profesional". Universidad de Alcalá- España. $2008^{(111)}$ Coincidiendo en las aseveraciones del presente trabajo en que las concepciones epistemológicas priorizados en mayor proporción, son el cuidado individual, único, el proceso interactivo enfermerapaciente.

\section{Valoración Del Ejercicio Profesional.}

En el trabajo Francisco Del Rey, Cristina Respecto a la valoración de la práctica profesional, se observa la existencia de un buen nivel de satisfacción con su ejercicio $(87,3 \%)$ y de la valoración por parte de los clientes $(60,5 \%)$. La satisfacción es relativa cuando se valora la percepción que se tiene de la relación entre la formación y su aplicación en la práctica $(49,4 \%)$ y de la propia enfermería $(36,2 \%)$. Y se observa insatisfacción en relación a la valoración que se hace desde los medios de comunicación $(53,9 \%)$, por otros profesionales $(42,4 \%)$ y por la población en general, que no ha sido cliente de sus servicios $(29,6 \%)^{(12)}$. Coincidiendo a las conclusiones del presente trabajo en cuanto al ejercicio profesional, $52.5 \%$ están en muy satisfechos.

\section{CONCLUSIONES}

1. Los docentes se caracterizan por tener edad promedio 58.6 años, grupo etario prevalente 52-60 años (40\%), mayoritariamente proceden de universidades estatales, $65 \%$ tienen especialidad, $87.5 \%$ son magísteres, $32.5 \%$ doctores. Ejercicio profesional promedio 31 años, docente 21 años; 47.5\% son docentes principales, $45 \%$ asociados, laboran en diversos servicios de hospitales de EsSalud y MINSA.

2. En cuanto a su formación profesional, $85 \%$ decidieron ser enfermeros porque les gustaba la carrera, $65 \%$ por vocación, $10 \%$ siguieron la tradición familia. Mayoritariamente consideran suficiente la 
formación teórica y práctica que recibieron. Aplican modelos teóricos y conceptuales de enfermería, clasifican NANDA, NOC, NIC, planes de cuidado. Elaboran - publican trabajos sobre cuidados técnicos, y de satisfacción de usuarios. Consideran que el rol autónomo es característico de la profesión.

3. Las concepciones epistemológicas priorizados en mayor proporción, son el cuidado individual, único, el proceso interactivo enfermera-paciente, la satisfacción de las necesidades de las personas, la concepción integral y sistémica del paciente: Física-asistencial-emocionalcultural-espiritual.

4. En cuanto al ejercicio profesional, $52.5 \%$ están en muy satisfechos, $35 \%$ satisfechos; señalan que desde el área asistencial se realizan las aportaciones más significativas para la profesión (47.5\%), y en menor medida del área de investigación (27.5\%) y docencia (17.5\%). Mayoritariamente utilizan proceso de atención de enfermería, y con frecuencia paradigmas epistemológicos.

\section{REFERENCIAS BIBLIOGRÁFICAS}

Navarro G. Epistemología, ciencia y disciplina. Lima, Perú 2015 [Internet]. Disponible en: http://epistemologiaenmiexperienciadocente. blogspot.pe/

Gutierrez L. Formación profesional factor determinante en el ejercicio profesional con calidad en enfermería. Rev Enferm Inst Mex Seguro Soc. 2008 Sept-Dic; 16(3):121-25

González E, Arras M, Moriel L. La profesionalización en enfermería: hacia una estrategia de cambio. Tecnociencia Chihuaha. 2012 Ene-Abr; 6(1):1-8.

Ariza C. Desarrollo epistemológico de enfermería. Enferm. univ . 2011, vol.8, n.2, pp.18-24. ISSN 2395-8421.
Arroyo J. Cohabitación o calidad de la formación: Dilema en los Campos Clínicos. 1a. Edición, Lima: IDREH-Proyecto AMARES, 2011. Pp. 38

Verástegui M. “Significado Del Cuidado De Enfermería Otorgado Por Las Enfermeras De Un Servicio De Cirugía De Un Hospital De Chiclayo 2012" Chiclayo.2014.

Oxford Dictionaries. Epistemology. Electronic Citation; 2010. Disponible en: http:// oxforddictionaries.com/definition/english/ epistemology

[Consultado el 10 de diciembre de 2010]

Dickoff J, James P. A theory of theories: A position paper. Nursing Research 1968; 17 (3): 19720.

Estrada E. Aplicación Del Proceso Atención De Enfermería En México: Un Análisis Epistemológico [Tesis Para Obtener El Grado De: Maestra En Enfermería Con Opción Terminal En Oncología]. Toluca, México .2014.Pp. 141-143.

Francisco C. De La Práctica De La Enfermería A La Teoría Enfermera. Concepciones Presentes En El Ejercicio Profesional. Universidad de Alcalá- España. 2008. Pp. 231,240-241.

Estrada E. Aplicación Del Proceso Atención De Enfermería En México: Un Análisis Epistemológico [Tesis Para Obtener El Grado De: Maestra En Enfermería Con Opción Terminal En Oncología]. Toluca, México .2014.Pp. 191

Francisco C. De La Práctica De La Enfermería A La Teoría Enfermera. Concepciones Presentes En El Ejercicio Profesional. Universidad de Alcalá- España. 2008. Pp. 252 


\section{ANEXOS}

Gráfico 1. Decisión de estudiar enfermería

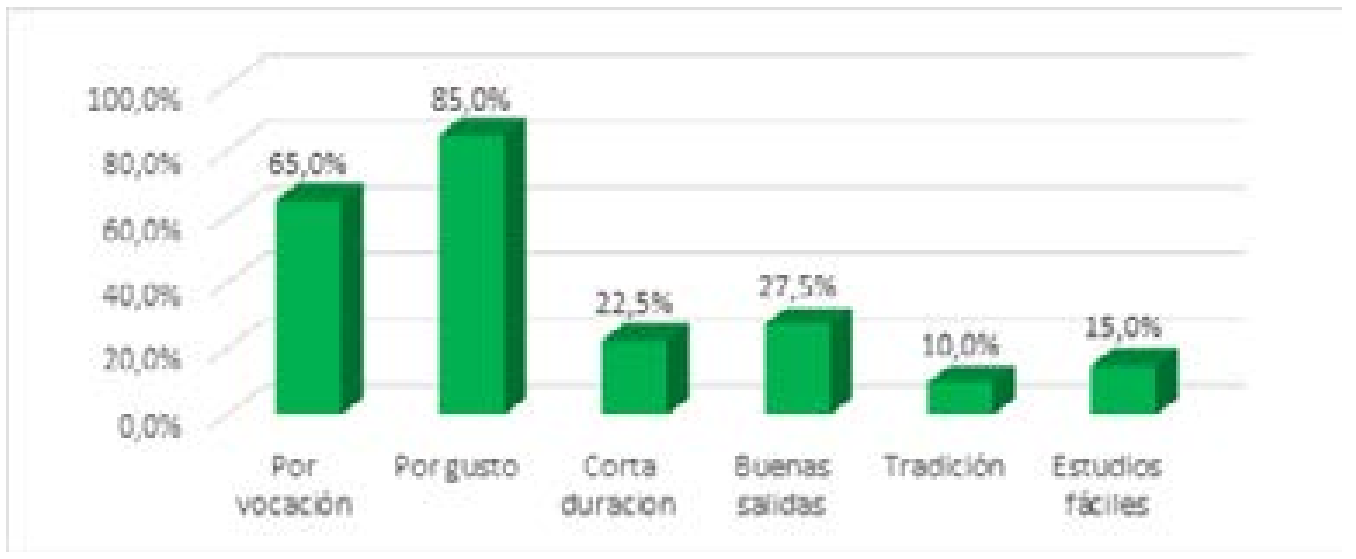

Gráfico2. Satisfacción con el ejercicio profesional

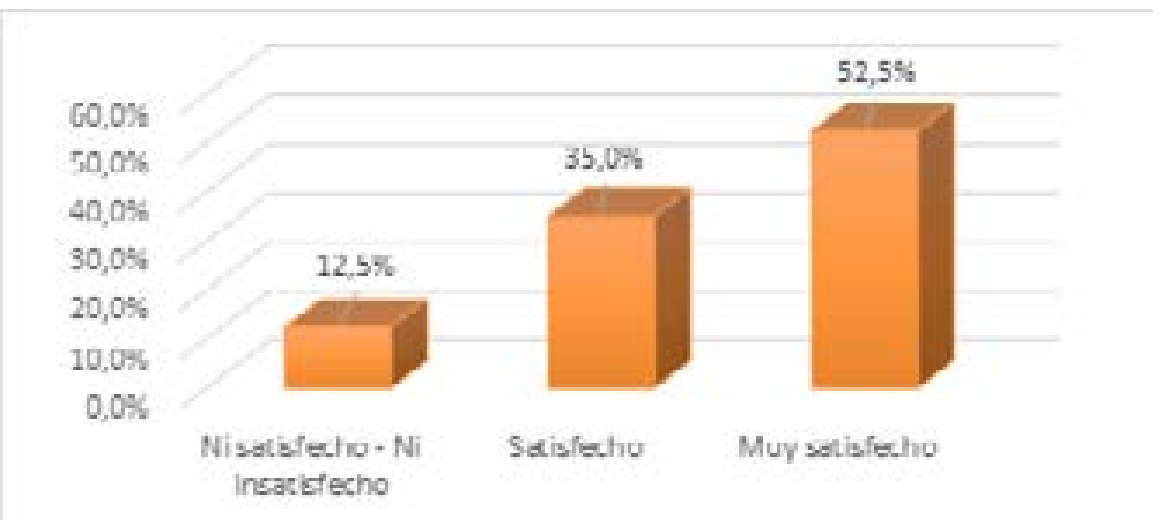

